\title{
Zitterbewegung of electrons in graphene in a magnetic field
}

\author{
Tomasz M. Rusin** and Wlodek Zawadzki ${ }^{*}$ \\ *PTK Centertel Sp. z o.o., ul. Skierniewicka 10A, 01-230 Warsaw, Poland \\ $\dagger$ Institute of Physics, Polish Academy of Sciences, Al. Lotników 32/46, 02-688 Warsaw, Poland
}

(Dated: July 6, 2018)

\begin{abstract}
Electric current and spacial displacement due to trembling motion [Zitterbewegung (ZB)] of electrons in graphene in the presence of an external magnetic field are described. Contributions of both inequivalent $K$ points in the Brillouin zone of graphene are considered. It is shown that, when the electrons are prepared in the form of wave packets, the presence of a quantizing magnetic field $B$ has very important effects on ZB. (1) For $B \neq 0$ the $\mathrm{ZB}$ oscillations are permanent, for $B=0$ they are transient. (2) For $B \neq 0$ many $\mathrm{ZB}$ frequencies appear, for $B=0$ only one frequency is at work. (3) For $B \neq 0$ both interband and intraband (cyclotron) frequencies contribute to $\mathrm{ZB}$, for $B=0$ there are no intraband frequencies. (4) Magnetic field intensity changes not only the ZB frequencies but the entire character of ZB spectrum. An emission of electromagnetic dipole radiation by the trembling electrons is proposed and described. It is argued that graphene in a magnetic field is a promising system for an experimental observation of Zitterbewegung.

PACS numbers: 73.22.-f, 73.63.Fg, 78.67.Ch, 03.65.Pm
\end{abstract}

\section{INTRODUCTION}

The Zitterbewegung (ZB, trembling motion), first described by Schrodinger with the use of the Dirac equation for free relativistic electrons in a vacuum [1], has been in recent years a subject of great theoretical interest since it has been shown that this phenomenon should exist in many systems in solids $[2,3,4,45,6,6,8,6,10,11,12,13$. . If one deals with two or more interacting energy branches, an interference of the upper and lower energy states gives rise to the $\mathrm{ZB}$ also in the absence of external fields. A formal similarity between two bands interacting via the $\mathbf{k} \cdot \mathbf{p}$ terms in a solid and the Dirac equation for relativistic electrons in a vacuum allows one to apply similar theoretical methods for both systems (see [14, 15]). Most of the theoretical ZB treatments for semiconductors used as a starting point plane electron waves. However, Lock [16] in his important paper observed that, since such a wave is not localized, it seems of a limited practicality to speak of rapid oscillations on the average position of a wave of infinite extent. Using the Dirac equation Lock demonstrated that, when an electron is represented by a wave packet, the ZB oscillations do not sustain their amplitude but become transient. The disappearance of oscillations at large times is guaranteed by the Riemann-Lebesgues theorem as long as the wave packet is a smoothly varying spatial function. The transient character of the trembling motion was demonstrated in our recent papers [12, 17]. Since the ZB is by its nature not a stationary state but a dynamical phenomenon, it is natural to study it with the use of wave packets (see [18]). These have become of practical use with the emergence of the femto-second pulse technology [19]. Recently, the transient trembling motion was proposed for ultra cold atoms [20, 21], for

*Electronic address: Tomasz.Rusin@centertel.pl photons in two-dimensional photonic crystals 22] and for Ramsey interferometry [23]. Most recently, an actual $o b$ servation of an acoustic analogue of $\mathrm{ZB}$ in a macroscopic two-dimensional sonic crystal was reported [24].

The trembling motion of charge carriers in solids has been described until present for no external potentials. On the other hand, Lock has remarked that, when an electron spectrum is discrete, the $\mathrm{ZB}$ oscillations do not have to disappear with time. In the present work we consider the trembling motion of electrons in solids in the presence of an external magnetic field, see [25, 26]. The magnetic field is known to cause no interband electron transitions, so the essential features of $\mathrm{ZB}$, which results from an interference of positive and negative energy states of the system, are expected not to be destroyed. On the other hand, introduction of an external field provides an important parameter affecting the $\mathrm{ZB}$ behavior. We show that, indeed, the $\mathrm{ZB}$ in a magnetic field is not damped in time.

We consider the ZB phenomenon in monolayer graphene. This material has recently become an important subject of investigation in the condensed matter physics and its applications [27, 28, 29]. In particular, the charge carriers in graphene are considered to represent massless Dirac fermions. In our approach we look for observable ZB phenomena. The situation we describe seems to be the most promising case for an experimental observation of the trembling motion in solids considered until present.

The paper is organized as follows. First, we consider the Hamiltonian for electrons in graphene in a magnetic field, its eigenvalues and eigenfunctions. Second, we calculate carrier velocities and their averages taken over a Gaussian wave packet. Third, we give results for the ZB of electric current and electron position and emphasize the essential features introduced by the presence of a magnetic field. Finally, we propose and describe electromagnetic radiation emitted by the trembling electrons. 
The paper is concluded by a summary. In Appendices we discuss additional points related to the subject.

\section{PRELIMINARIES}

We consider a graphene monolayer in an external magnetic field parallel to the $z$ axis. As shown in both continuum and tight-binding models, there exist two inequivalent points $K_{1}$ and $K_{2}$ of the Brillouin zone (BZ) of graphene. The Hamiltonian for electrons and holes at the $K_{1}$ point is 30,31 .

$$
\hat{H}=u\left(\begin{array}{cc}
0 & \hat{\pi}_{x}-i \hat{\pi}_{y} \\
\hat{\pi}_{x}+i \hat{\pi}_{y} & 0
\end{array}\right)
$$

where $u \approx 1 \times 10^{8} \mathrm{~cm} / \mathrm{s}$ is a characteristic velocity, $\hat{\pi}=$ $\hat{\boldsymbol{p}}-q \hat{\boldsymbol{A}}$ is the generalized momentum, $\hat{\boldsymbol{A}}$ is the vector potential and $q$ is the electron charge. We remark that the above Hamiltonian is not symmetric in $\hat{p}_{x}$ and $\hat{p}_{y}$. Using the Landau gauge we take $\hat{\boldsymbol{A}}=(-B y, 0,0)$, and for an electron $q=-e$ with $e>0$. Since in the above gauge $\hat{H}$ does not depend on $x$, we take the wave function in the form

$$
\Psi(x, y)=e^{i k_{x} x} \Phi(y)
$$

Introducing the magnetic radius $L=\sqrt{\hbar / e B}$ and the variable $\xi=y / L-k_{x} L$, we have

$$
\hat{H}=\frac{u \hbar}{L}\left(\begin{array}{cc}
0 & -\xi-\partial / \partial \xi \\
-\xi+\partial / \partial \xi & 0
\end{array}\right) .
$$

Defining the standard raising and lowering operators for the harmonic oscillator $\hat{a}=(\xi+\partial / \partial \xi) / \sqrt{2}$ and $\hat{a}^{+}=$ $(\xi-\partial / \partial \xi) / \sqrt{2}$, the Hamiltonian becomes

$$
\hat{H}=-\hbar \Omega\left(\begin{array}{cc}
0 & \hat{a} \\
\hat{a}^{+} & 0
\end{array}\right)
$$

where the frequency is $\Omega=\sqrt{2} u / L$.

Next we determine the eigenstates and eigenenergies of the Hamiltonian $\hat{H}$. Using a two-component function $\psi=\left(\psi_{1}, \psi_{2}\right)$, we have

$$
\left\{\begin{aligned}
-\hbar \Omega \hat{a} \psi_{2} & =E \psi_{1} \\
-\hbar \Omega \hat{a}^{+} \psi_{1} & =E \psi_{2}
\end{aligned}\right.
$$

Expressing $\psi_{1}$ by $\psi_{2}$ from the first equation we obtain from the second equation: $\hbar^{2} \Omega^{2} \hat{a}^{+} \hat{a} \psi_{2}=E^{2} \psi_{2}$. The solution is $\psi_{2}=|n\rangle$, where $|n\rangle$ is the $n$-th state of the harmonic oscillator, and the energy is

$$
E_{n s}=s \hbar \Omega \sqrt{n} .
$$

Here $n=0,1, \ldots$, and $s= \pm 1$ for the conduction and valence bands, respectively. Formula (6) was experimentally confirmed for graphene in many works [28, 32, 33]. The functions $\langle\boldsymbol{r} \mid n\rangle$ are given by

$$
\langle\boldsymbol{r} \mid n\rangle=\frac{1}{\sqrt{L}} \frac{1}{C_{n}} e^{-\frac{1}{2} \xi^{2}} \mathrm{H}_{n}(\xi),
$$

where $C_{n}=\sqrt{2^{n} n ! \sqrt{\pi}}$, and $\mathrm{H}_{n}(\xi)$ are the Hermite polynomials. From Eq. (5) we obtain

$$
\psi_{1}=-\frac{\hbar \Omega \hat{a} \psi_{2}}{E}=-\frac{\hbar \Omega \sqrt{n}|n-1\rangle}{s \hbar \Omega \sqrt{n}}=-s|n-1\rangle .
$$

Each eigenstate $|n\rangle$ is labelled by three quantum numbers: oscillator number $n$, energy branch $s$, and wave vector $k_{x}$. The complete function is

$$
|\mathrm{n}\rangle \equiv\left|n k_{x} s\right\rangle=\frac{e^{i k_{x} x}}{\sqrt{4 \pi}}\left(\begin{array}{c}
-s|n-1\rangle \\
|n\rangle
\end{array}\right) .
$$

For $n=0$, the first component in Eq. (9) vanishes and the normalization coefficient is $1 / \sqrt{2 \pi}$.

\section{VELOCITIES. ZITTERBEWEGUNG}

We want to calculate the velocity of charge carriers described by a wave packet. In order to do that we first calculate matrix elements $\langle f \mid \mathrm{n}\rangle$ between an arbitrary twocomponent function $f=\left(f^{u}, f^{l}\right)$ and eigenstates (9). A straightforward manipulation gives

$$
\langle f \mid \mathrm{n}\rangle=-s F_{n-1}^{u}+F_{n}^{l},
$$

where

$$
F_{n}^{j}\left(k_{x}\right)=\frac{1}{\sqrt{2 L} C_{n}} \int g^{j}\left(k_{x}, y\right) e^{-\frac{1}{2} \xi^{2}} \mathrm{H}_{n}(\xi) d y,
$$

in which

$$
g^{j}\left(k_{x}, y\right)=\frac{1}{\sqrt{2 \pi}} \int f^{j}(x, y) e^{i k_{x} x} d x .
$$

The superscript $j=u, l$ stands for the upper and lower components of the function $f$.

The Hamilton equations give the velocity components: $\hat{v}_{i}(0)=\partial \hat{H} / \partial \hat{\pi}_{i}$, with $i=x, y$. We want to calculate averages of the time-dependent velocity operators $\hat{v}_{i}(t)$ in the Heisenberg picture taken on the function $f$. The averages are

$$
\bar{v}_{i}(t)=\sum_{\mathrm{n}, \mathrm{n}^{\prime}} e^{i E_{\mathrm{n}^{\prime}} t / \hbar}\left\langle f \mid \mathrm{n}^{\prime}\right\rangle\left\langle\mathrm{n}^{\prime}\left|v_{i}(0)\right| \mathrm{n}\right\rangle\langle\mathrm{n} \mid \mathrm{f}\rangle \mathrm{e}^{-\mathrm{i} E_{\mathrm{n}} \mathrm{t} / \hbar},
$$

where the energies and eigenstates are given in Eqs. (6) and (9). The summation in Eq. (13) goes over all the quantum numbers

$$
\sum_{\mathrm{n}, \mathrm{n}^{\prime}} \rightarrow \iint d k_{x} d k_{x}^{\prime} \sum_{n, n^{\prime}} \sum_{s, s^{\prime}}
$$

We calculate a contribution to the velocity from the point $K_{1}$ of the Brillouin zone. The matrix elements $\left\langle\mathrm{n}^{\prime}\left|v_{y}(0)\right| \mathrm{n}\right\rangle$ and $\left\langle\mathrm{n}^{\prime}\left|v_{x}(0)\right| \mathrm{n}\right\rangle$ can be shown to be

$$
\left\langle\mathrm{n}^{\prime}\left|v_{y}(0)\right| \mathrm{n}\right\rangle=\frac{i u}{2} \delta_{k_{x}, k_{x}^{\prime}}\left(-s \delta_{n^{\prime}, n-1}+s^{\prime} \delta_{n^{\prime}, n+1}\right),
$$




$$
\left\langle\mathrm{n}^{\prime}\left|v_{x}(0)\right| \mathrm{n}\right\rangle=-\frac{u}{2} \delta_{k_{x}, k_{x}^{\prime}}\left(s \delta_{n^{\prime}, n-1}+s^{\prime} \delta_{n^{\prime}, n+1}\right)
$$

It is seen that the only non-vanishing matrix elements of the velocity components are those with the final states $n^{\prime}=n \pm 1$. Putting the above matrix elements into Eq. (13), we finally obtain after some manipulation for the $K_{1}$ point of $\mathrm{BZ}$

$$
\begin{aligned}
& \bar{v}_{y}(t)=u \sum_{n=0}^{\infty} V_{n}^{+} \sin \left(\omega_{n}^{c} t\right)+u \sum_{n=0}^{\infty} V_{n}^{-} \sin \left(\omega_{n}^{Z} t\right)+i u \sum_{n=0}^{\infty} A_{n}^{+} \cos \left(\omega_{n}^{c} t\right)+i u \sum_{n=0}^{\infty} A_{n}^{-} \cos \left(\omega_{n}^{Z} t\right) \\
& \bar{v}_{x}(t)=u \sum_{n=0}^{\infty} B_{n}^{+} \cos \left(\omega_{n}^{c} t\right)+u \sum_{n=0}^{\infty} B_{n}^{-} \cos \left(\omega_{n}^{Z} t\right)+i u \sum_{n=0}^{\infty} T_{n}^{+} \sin \left(\omega_{n}^{c} t\right)+i u \sum_{n=0}^{\infty} T_{n}^{-} \sin \left(\omega_{n}^{Z} t\right)
\end{aligned}
$$

where

$$
\begin{aligned}
& V_{n}^{ \pm}=\mp U_{n-1, n}^{u, u} \mp U_{n, n-1}^{u, u}-U_{n+1, n}^{l, l}-U_{n, n+1}^{l, l}, \\
& T_{n}^{ \pm}= \pm U_{n-1, n}^{u, u} \mp U_{n, n-1}^{u, u}+U_{n+1, n}^{l, l}-U_{n, n+1}^{l, l}, \\
& A_{n}^{ \pm}=-U_{n, n}^{u, l}+U_{n, n}^{l, u} \pm U_{n-1, n+1}^{u, l} \mp U_{n+1, n-1}^{l, u}, \\
& B_{n}^{ \pm}=U_{n, n}^{u, l}+U_{n, n}^{l, u} \pm U_{n-1, n+1}^{u, l} \pm U_{n+1, n-1}^{l, u},
\end{aligned}
$$

in which

$$
U_{m, n}^{\alpha, \beta}=\int F_{m}^{\alpha *}\left(k_{x}\right) F_{n}^{\beta}\left(k_{x}\right) d k_{x}
$$

The superscripts $\alpha, \beta$ refer to the upper and lower components, see Eqs. (10). The velocity averages must be real values. For example, if both $g^{j}\left(k_{x}, y\right)(j=u, l)$ in Eq. (12) are real then also both $F_{n}^{j}\left(k_{x}\right)$ given by Eq. (11) are real, and there is $U_{m, n}^{\alpha, \beta}=U_{n, m}^{\beta, \alpha}$. As a result the last two terms in Eqs. (17) and (18) vanish.

The time dependent sine and cosine functions come from the exponential terms in Eq. (13). The frequencies in Eqs. (17) and (18) are $\omega_{n}^{c}=\Omega(\sqrt{n+1}-\sqrt{n})$, $\omega_{n}^{Z}=\Omega(\sqrt{n+1}+\sqrt{n})$, where $\Omega$ is given in Eq. (44). The frequencies $\omega_{n}^{c}$ correspond to the intraband (cyclotron) energies while frequencies $\omega_{n}^{Z}$ correspond to the interband energies, see Fig. 1. The interband frequencies are characteristic of the Zitterbewegung because the trembling motion is caused by an interference of states corresponding to the positive and negative energies [34, 35]. The intraband (cyclotron) energies are due to the band quantization by the magnetic field and they do not appear in field-free situations (see [4, 5, 6, 12]).

\section{GAUSSIAN WAVE PACKET}

We take the function $f(x, y)$ in the form of a Gaussian wave packet having an initial nonvanishing momentum $p_{0 x}=\hbar k_{0 x}$

$$
f(x, y)=\frac{1}{\sqrt{\pi d_{x} d_{y}}} e^{-\frac{x^{2}}{2 d_{x}^{2}}-i k_{0 x} x-\frac{y^{2}}{2 d_{y}^{2}}}\left(\begin{array}{c}
a_{u} \\
a_{l}
\end{array}\right) .
$$

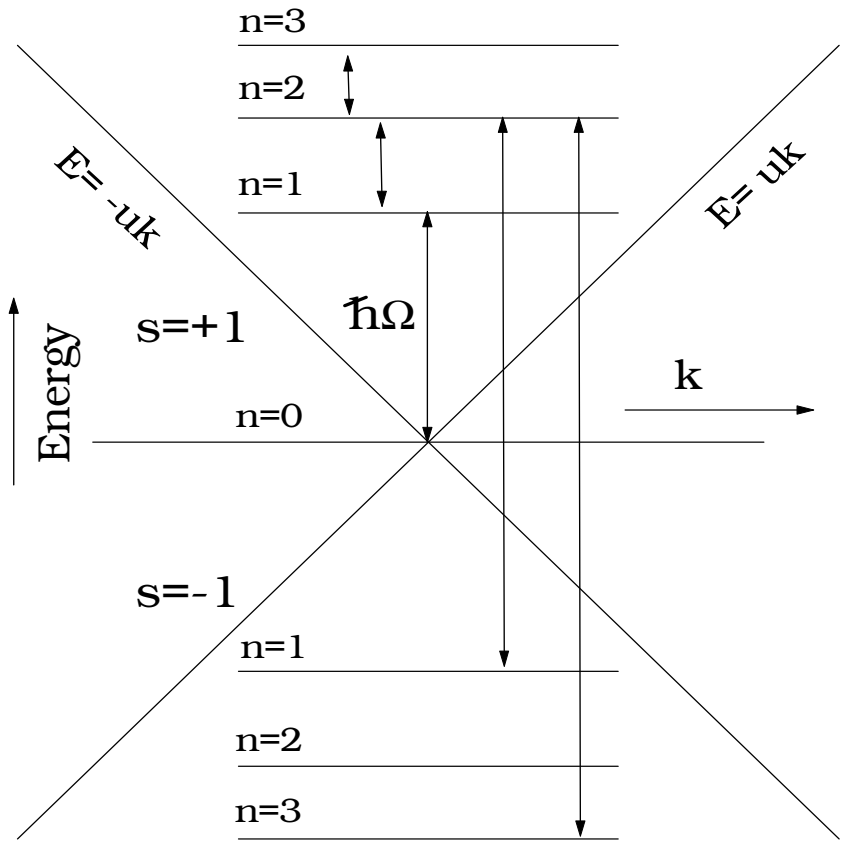

FIG. 1: The energy dispersion $E(k)$ and the Landau levels for graphene in a magnetic field (schematically). Intraband (cyclotron) and interband energies for $n^{\prime}=n \pm 1$ are indicated, see text. The basic energy is $\hbar \Omega=\sqrt{2} \hbar u / L$.

In the above model the upper and lower components of $f$ differ only by the coefficients $a_{u}$ and $a_{l}$, respectively. There is $a_{u}^{2}+a_{l}^{2}=1$. Then [see Eq. (12)]

$$
g\left(k_{x}, y\right)=\sqrt{\frac{d_{x}}{\pi d_{y}}} e^{-\frac{1}{2} d_{x}^{2}\left(k_{x}-k_{0 x}\right)^{2}} e^{-\frac{y^{2}}{2 d_{y}^{2}}}\left(\begin{array}{c}
a_{u} \\
a_{l}
\end{array}\right)
$$

This gives [see Eq. (11)]

$$
F_{n}^{\alpha}\left(k_{x}\right)=\frac{a_{\alpha} A_{n} \sqrt{L d_{x}}}{\sqrt{2 \pi d_{y}} C_{n}} e^{-\frac{1}{2} d_{x}^{2}\left(k_{x}-k_{0 x}\right)^{2}} e^{-\frac{1}{2} k_{x}^{2} D^{2}} \mathrm{H}_{n}\left(k_{x} c\right)
$$


Here $D=L^{2} / \sqrt{L^{2}+d_{y}^{2}}, c=L^{3} / \sqrt{L^{4}-d_{y}^{4}}$, and

$$
A_{n}=\frac{\sqrt{2 \pi} d_{y}}{\sqrt{L^{2}+d_{y}^{2}}}\left(\frac{L^{2}-d_{y}^{2}}{L^{2}+d_{y}^{2}}\right)^{n / 2}
$$

After some manipulations, we finally find (see [36])

$$
\begin{gathered}
U_{m, n}^{\alpha, \beta}=\frac{a_{\alpha} a_{\beta} A_{m}^{*} A_{n} L Q d_{x} \sqrt{\pi} e^{-W^{2}}}{2 \pi C_{m} C_{n} d_{y}} \sum_{l=0}^{\min \{m, n\}} 2^{l} l !\left(\begin{array}{c}
m \\
l
\end{array}\right)\left(\begin{array}{c}
n \\
l
\end{array}\right) \\
\quad \times\left(1-(c Q)^{2}\right)^{(m+n-2 l) / 2} \mathrm{H}_{m+n-2 l}\left(\frac{c Q Y}{\sqrt{1-(c Q)^{2}}}\right),
\end{gathered}
$$

where $Q=1 / \sqrt{d_{x}^{2}+D^{2}}, W=d_{x} D Q$, and $Y=d_{x}^{2} k_{0 x} Q$. Thus in case of a Gaussian wave packet we obtain the coefficients $U_{m, n}^{\alpha, \beta}$ in the form of analytical sums. For the special case $d_{y}=L$, there is simply

$$
\begin{aligned}
U_{m, n}^{\alpha, \beta}= & \frac{\sqrt{\pi} i^{m+n} a_{\alpha} a_{\beta} d_{x}}{C_{m} C_{n} L}\left(\frac{L}{2 P}\right)^{m+n+1} \times \\
& \exp \left(-\frac{d_{x}^{2} k_{0 x}^{2} L^{2}}{2 P^{2}}\right) \mathrm{H}_{m+n}\left(\frac{-i d_{x}^{2} k_{0 x}}{P}\right),
\end{aligned}
$$

where $P=\sqrt{d_{x}^{2}+\frac{1}{2} L^{2}}$. In the above model the coefficients $U_{m, n}^{\alpha, \beta}$ are real numbers, so that $A_{n}^{ \pm}$and $T_{n}^{ \pm}$in Eqs. (17) and (18) vanish. A sum rule for $U_{n, n}^{\alpha, \beta}$ is given in Appendix A.

\section{RESULTS AND DISCUSSION}

In Fig. 2 we show calculated current components $j_{i}=-e \bar{v}(t)$ as functions of time for different values of the initial wave vector $k_{0 x}$. The calculations were carried out for the $K_{1}$ point of BZ taking $a_{u}=a_{l}=1 / \sqrt{2}$ and a constant magnetic field of $10 \mathrm{~T}$. We assumed a circular wave packet $d_{x}=d_{y}=L=83.13 \AA$, the coefficients $U_{m, n}^{\alpha, \beta}$ were calculated using Eq. (26). Figure 2a shows the results for $k_{0 x}=0$. It can been seen that, if there is no initial momentum, only $j_{x}(t)$ is nonzero. The main frequency of oscillations is $\omega_{0}=\Omega$, which can be interpreted either as $\omega_{0}^{c}=\Omega(\sqrt{n+1}-\sqrt{n})$ or $\omega_{0}^{Z}=\Omega(\sqrt{n+1}-\sqrt{n})$ for $n=0$. Frequency $\omega_{0}^{c}$ belongs to the intraband (cyclotron) set, while $\omega_{0}^{Z}$ belongs to the interband set (see Fig. 1). Somewhat unequal amplitude of oscillations means that other frequencies also come into play, so that we already deal with the trembling motion. For $k_{0 x}=0$, the asymmetry between $\bar{v}_{x}(t)$ and $\bar{v}_{y}(t)$ comes from the above mentioned asymmetry of the initial Hamiltonian with respect to $\hat{p}_{x}$ and $\hat{p}_{y}$, see Eq. (11). In Figs. 2b, $2 \mathrm{c}$ and $2 \mathrm{~d}$ we show calculated contributions to the current for growing values of $k_{0 x}$. For nonzero values of $k_{0 x}$, both $j_{x}$ and $j_{y}$ appear. It is seen that the frequency and the shape of ZB oscillations change with growing $k_{0 x}$ values. For growing $k_{0 x}$ different $U_{m, n}^{\alpha, \beta}$ become large and in

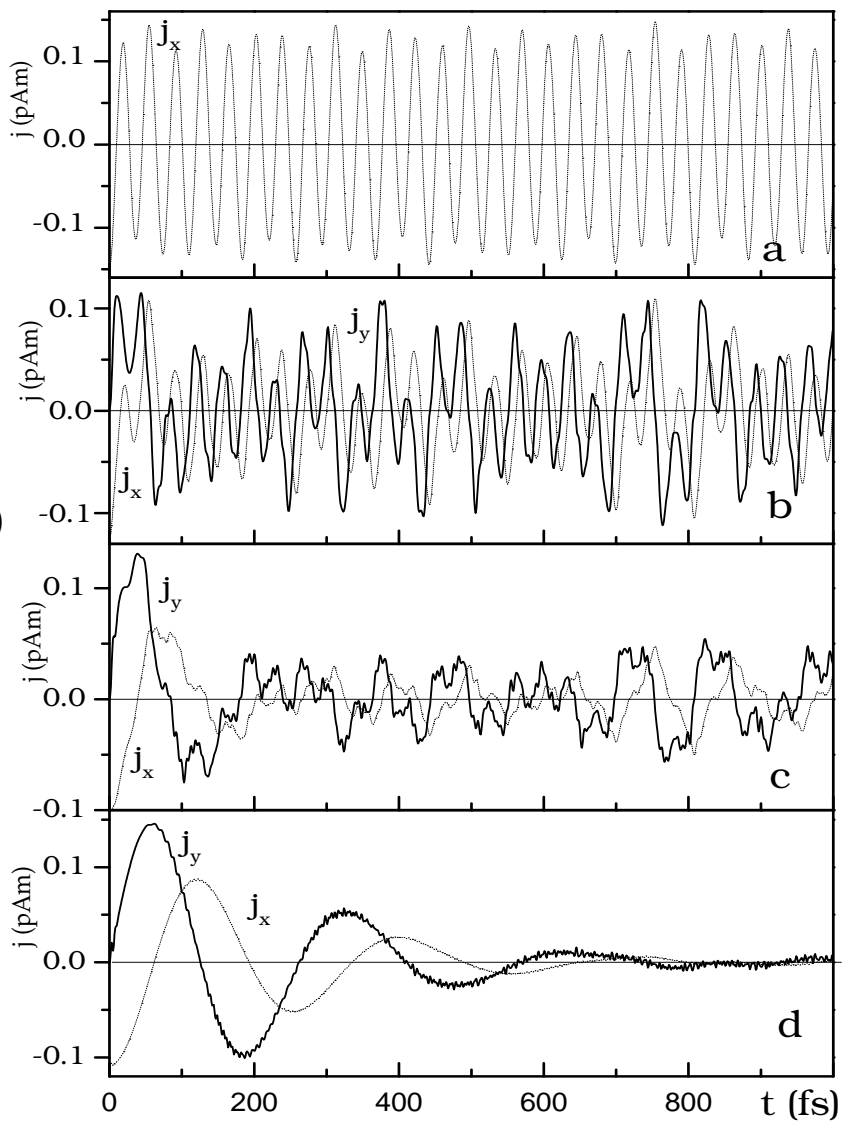

FIG. 2: Contribution of the $K_{1}$ point of the Brillouin zone to the electric current in graphene at $\mathrm{B}=10 \mathrm{~T}$ versus time, calculated for a Gaussian wave packet of the width $d_{x}=d_{y}=$ $L=81.13 \AA$ and various values of $k_{0 x}$ : a) $k_{0 x}=0$, b) $k_{0 x}=$ $0.02 \AA^{-1}$, c) $k_{0 x}=0.035 \AA^{-1}$, d) $k_{0 x}=0.06 \AA^{-1}$. Thick lines $-j_{y}(t)$, thin lines $-j_{x}(t)$.

consequence different frequencies $\omega_{n}^{c}$ and $\omega_{n}^{Z}$ dominate in sums (17) and (18). The striking feature seen in Figs. $2 \mathrm{a}, 2 \mathrm{~b}$ and $2 \mathrm{c}$ is, that the $\mathrm{ZB}$ is manifested by several frequencies simultaneously. This is a consequence of the fact that, as follows from Eq. (6), in graphene the energy distances between the Landau levels diminish with $n$, which results in different values of frequencies $\omega_{n}^{c}$ and $\omega_{n}^{Z}$ for different $n$. Thus, it is the presence of an external quantizing magnetic field that introduces various frequencies into ZB.

For sufficiently large values of $k_{0 x}$ only one frequency prevails, as shown in Fig. 2d. This is related to the fact that, as seen in the inset of Fig. 8 in Appendix B, the coefficients $U_{m, n}^{\alpha, \beta}$ in this regime have a pronounced maximum around a specific value $n_{\max }$. The dominant frequency is $\omega_{\max }=\omega\left(\sqrt{n_{\max }+1}-\sqrt{n_{\max }}\right)$, which is simply the cyclotron frequency for $n_{\max }$, see Fig. 1. Thus, it might appear that the current shown in Fig. 2d corresponds to the simple classical cyclotron motion and the trembling motion is manifested only by the damping in time (see Appendix D). This is however not the case.

In Fig. 3 we show the calculated current for $k_{0 x}=$ 


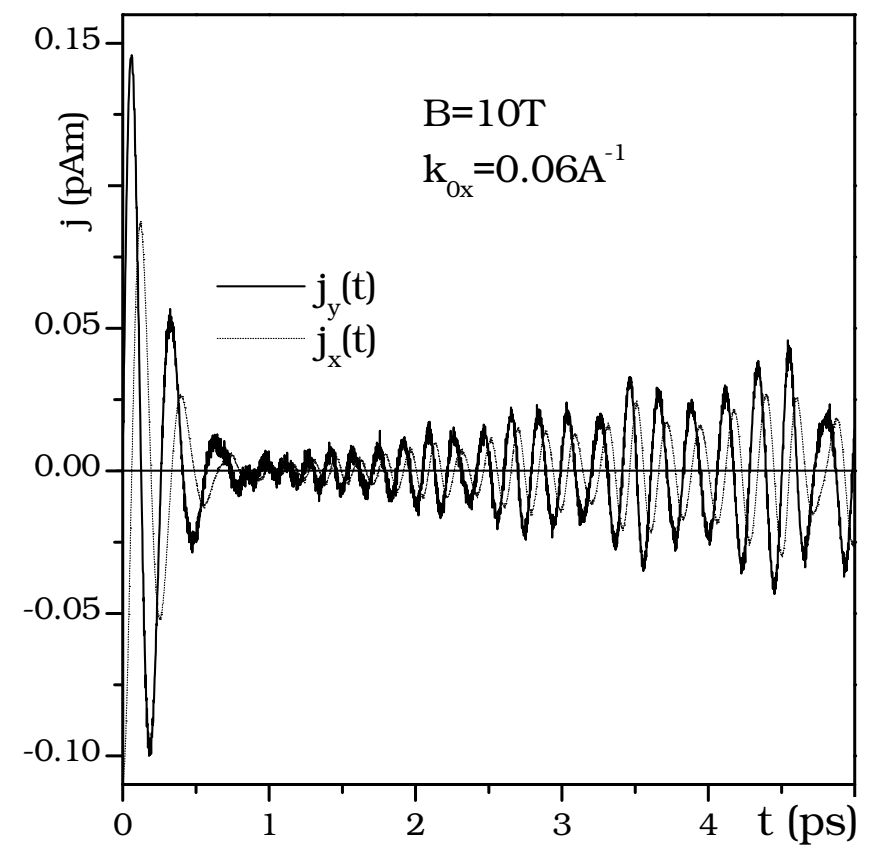

FIG. 3: The same as Fig. 2d, but for larger time scale. Results for the first picosecond coincide with those shown in Fig. 2d.

$0.06 \AA^{-1}$ (the same as in Fig. 2d), but in a much larger time scale. It turns out that, after the $\mathrm{ZB}$ oscillations seemingly die out, they actually reappear.

Thus, for all $k_{0 x}$ values (including $k_{0 x}=0$ ), the $Z B$ oscillations have a permanent character, that is they do not disappear in time. This feature is due to the discrete character of the electron spectrum caused by a magnetic field. The above property is in sharp contrast to the no-field cases considered until present, in which the spectrum is not quantized and the ZB of a wave packet has a transient character, see [12, 17]. In mathematical terms, due to the discrete character of the spectrum, averages of operator quantities taken over a wave packet are sums and not integrals, see Eqs. (17) and (18). The sums do not obey the Riemann-Lebesgues theorem for integrals which guaranteed the damping of $\mathrm{ZB}$ in time for a continuous spectrum (see Ref. [16]). We consider the demonstration of a permanent character of ZB oscillations for a discrete spectrum to be the main result of our present work.

In Fig. 4 we show the $\mathrm{ZB}$ oscillations of the current for a constant wave vector $k_{0 x}=0.035 \AA^{-1}$ at different magnetic fields. It can be seen that the intensity of a magnetic field has a dramatic effect on ZB: not only its frequency is changed but also the character of oscillations. Lower magnetic fields are equivalent to higher $k_{0 x}$ values, since both lead to higher Landau levels involved (see Figs. 2d and 4a). Inversely, higher magnetic fields and lower $k_{0 x}$ values lead to lower Landau levels involved (see Figs. 2b and 4c). At very small magnetic fields there exist three regimes of ZB oscillations: the 'initial' oscil-

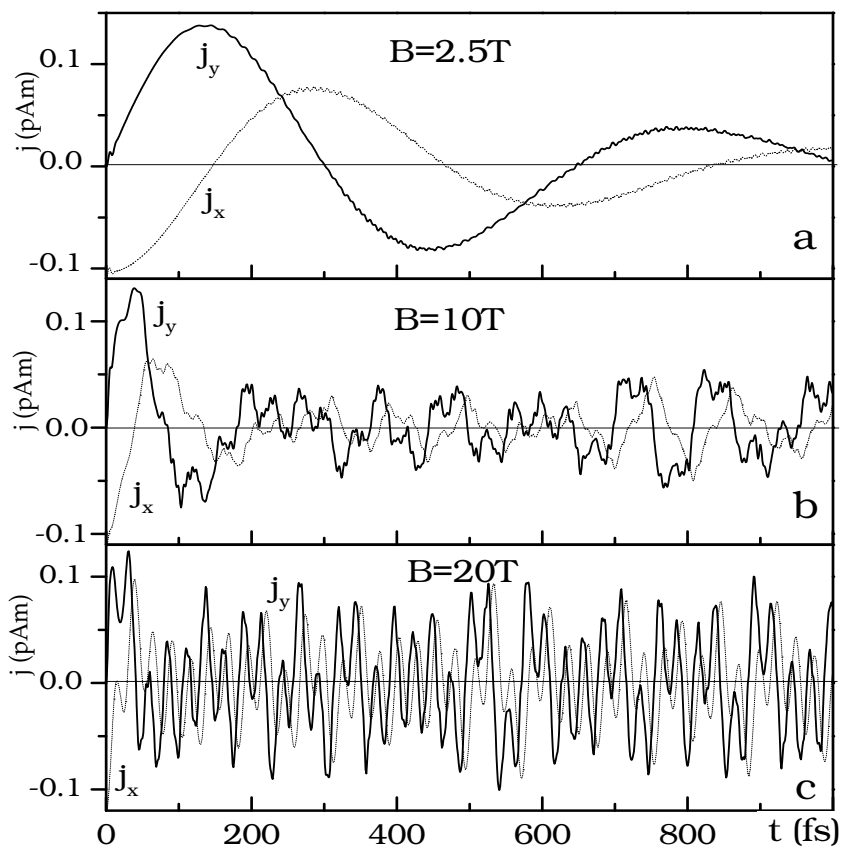

FIG. 4: The same as in Fig. 2, calculated for fixed values of $k_{0 x}=0.035 \AA^{-1}$ and widths $d_{x}=d_{y}=81.13 \AA$ but at different magnetic fields. Results $4 \mathrm{~b}$ are the same as those shown in Fig. 2d.

lations dying out during a few femtoseconds (as shown in Fig. 2 of Ref. [12]), the second range of oscillations dying out during several picoseconds, as shown in Fig. 2d, and the third range of permanent, somewhat irregular oscillations shown for $t \geq 1$ ps in Fig. 3 .

Finally, we calculate the displacements $\bar{x}(t)$ and $\bar{y}(t)$ of the wave packet. To this end we integrate expressions (17) and (18) with respect to time using the initial conditions $x_{0}=\bar{x}(0)$ and $y_{0}=\bar{y}(0)$. The results are plotted in Fig. 5 in the form of $x-y$ trajectories for different initial wave vectors $k_{0 x}$. The direction of movement is clockwise. The trajectories span early times (1ps) after the creation of a wave packet. As mentioned above, the $\mathrm{ZB}$ oscillations do not die out in time which is reflected by infinite trajectories. In Fig. 5 the trajectories are shown around the point $x_{0}=y_{0}=0$, whereas in reality the $y$ component of the center is almost a linear function of $k_{0 x}: y_{0} \approx k_{0 x} L^{2}$.

All in all, the presence of a quantizing magnetic field has the following important effects on the trembling motion. (1) For $B \neq 0$ the $\mathrm{ZB}$ oscillation are permanent, while for $B=0$ they are transient. (2) For $B \neq 0$ many ZB frequencies appear, whereas for $B=0$ only one ZB frequency exists. (3) For $B \neq 0$ both interband and intraband (cyclotron) frequencies appear in $\mathrm{ZB}$; for $B=0$ there are no intraband frequencies. (4) Magnetic field intensity changes not only the $\mathrm{ZB}$ frequencies but the entire character of ZB spectrum.

The results shown in Fig. 2 were obtained using the simplifying assumption about packet's width: $d_{x}=d_{y}=$ 


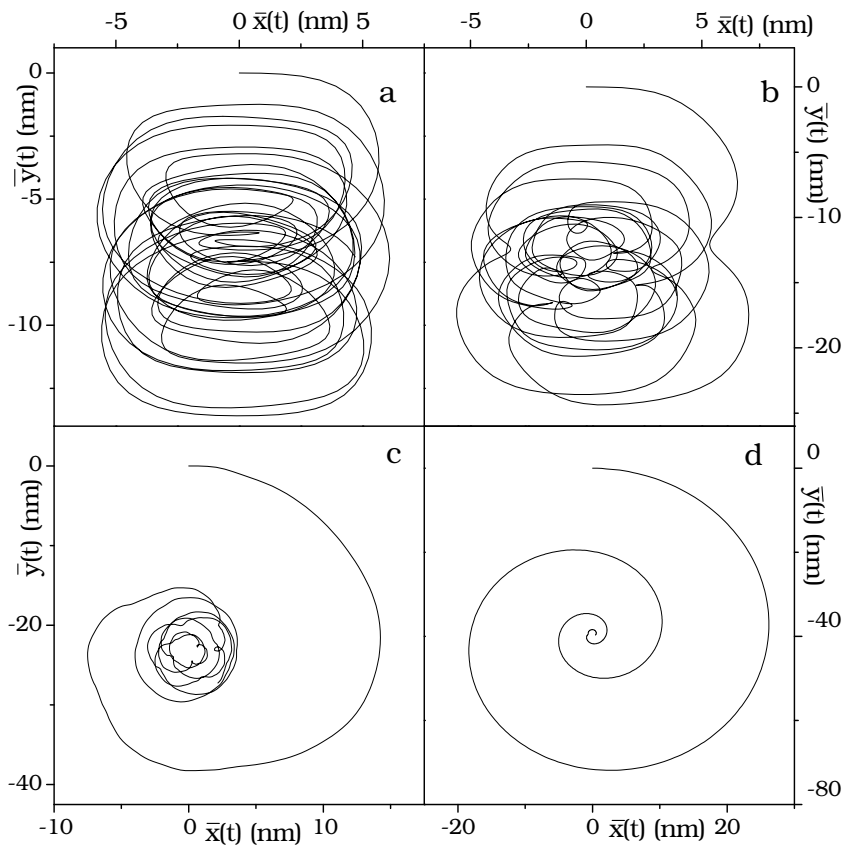

FIG. 5: Zitterbewegung trajectories of electron at the $K_{1}$ point of the Brillouin zone during the first picosecond for various values of $k_{0 x}$ : a) $k_{0 x}=0.01 \AA^{-1}$, b) $k_{0 x}=0.02 \AA^{-1}$ c), $\left.k_{0 x}=0.035 \AA^{-1} \mathrm{~d}\right), k_{0 x}=0.06 \AA^{-1}$.

$L=(\hbar / e B)^{1 / 2}$. This allowed us to use formula (26) for the calculation of $U_{m, n}^{\alpha, \beta}$. However, in Fig. 4 we show the results obtained for constant $d_{x}=d_{y}$ and variable $B$, for which we had to use general formula (25). In all the calculations involving magnetic field, precise numerical values of the Hermite polynomials are required, see Eqs. (25) and (26). For the results shown above we used the values of the first 400 Hermite polynomials, and checked their high precision using sum rule A1). We also considered the case of an initial electron momentum directed not along the $x$ direction (as shown above), but also along the $y$ direction. In this case the $U_{m, n}^{\alpha, \beta}$ coefficients are imaginary, so that only the last two terms of Eqs. (17) and (18) come into play. The final results are similar but not identical to those quoted above. The reason is that, as already mentioned, the initial Hamiltonian (11) is not symmetric in $\hat{p}_{x}$ and $\hat{p}_{y}$ momenta. When using Gaussian wave packet (21), we assumed equal upper and lower components $a_{u}=a_{l}=1 / \sqrt{2}$. This is in contrast to previous papers which usually took $a_{u}=1, a_{l}=0$ [4, 11, 12, 16, 18]. This choice is somewhat arbitrary, it is determined by an experimental wave packet usually prepared by optical methods. One should keep in mind that the relative final amplitudes of $\bar{v}_{x}(t)$ and $\bar{v}_{y}(t)$ (and the resulting currents) depend on this choice via $U_{m, n}^{\alpha, \beta}$ coefficients, see Eqs. (25) and (26). If one chooses $a_{u}=1$, $a_{l}=0$, the resulting motion coming from the $K_{1}$ point of $\mathrm{BZ}$ is only along the $y$ direction.

It is of interest that phenomena analogous to those described above for electrons, occur also for photons. In

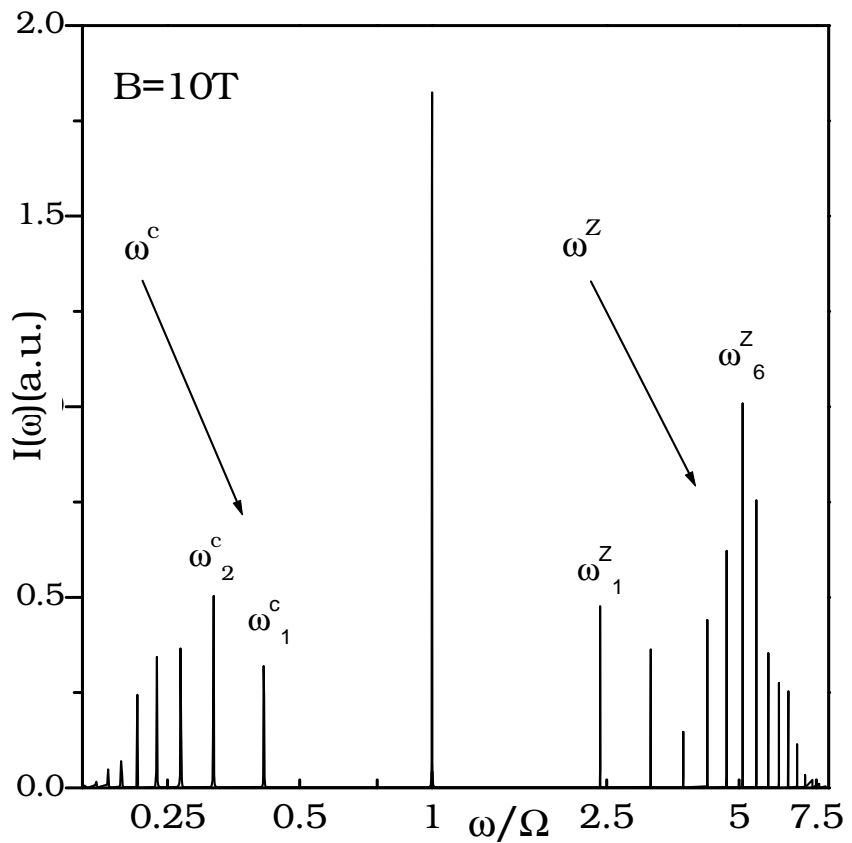

FIG. 6: Intensity spectrum versus frequency during the first 20 ps of motion of an electron described by a Gaussian wave packet having $k_{0 x}=0.035 \AA^{-1}$ at $B=10 \mathrm{~T}$. The frequencies $\omega_{n}^{c}$ and $\omega_{n}^{Z}$ are defined in Eqs. (17) and (18).

particular, Hamiltonian (4) is similar to that describing an interaction of atoms with electromagnetic radiation according to the so called Jaynes-Cummings model [37, 38]. In particular, the collapse and revival of electron's ZB oscillations, as illustrated in Figs. 20 and 3, is predicted by the Jaynes-Cummings model for the number of emitted photons ant is was observed in one-electron masers [39].

\section{DIPOLE RADIATION DUE TO ZB}

Experimental possibilities of observing the trembling motion were considered previously [4, 6, 12] and we do not consider this problem again. The results shown in Figs. 2, 3 and 4 describe the electric current, which is an observable quantity. One could also try to see directly the displacement of charge, cf. [40, 41]. On the other hand, we argue below that the ZB should be accompanied by electromagnetic dipole radiation emitted by the trembling electrons. The oscillations $\bar{r}(t)$, as shown in Fig. 5, are related to the dipole moment $-e \bar{r}(t)$, which couples to the electromagnetic radiation. We shall treat the radiation classically [42], i.e. we take the radiated transverse electric field to be [43]

$$
\mathcal{E}_{\perp}(\boldsymbol{r}, t)=\frac{e \overline{\overline{\boldsymbol{r}}}(t)}{4 \pi \epsilon_{0} c^{2}} \frac{\sin (\theta)}{R},
$$

where $\epsilon_{0}$ is the vacuum permittivity, $\theta$ is an angle between the direction of motion of a wave packet and the position 
of the observer $\boldsymbol{R}$. Integrating $\mathcal{E}_{\perp}^{2}$ over the angle $\theta$ one obtains the total radiated power given by the Larmor formula

$$
P=\frac{e^{2} \bar{a}^{2}(t)^{2}}{6 \pi \epsilon_{0} c^{3}},
$$

where $\bar{a}$ is the acceleration averaged over the packet. To find $P$ we calculate the acceleration from Eqs. (17) and (18) by taking the time derivatives. The spectrum of the emitted radiation is obtained by the Fourier transform of the electric field. We have

$$
\overrightarrow{\mathcal{E}}(t)=\frac{1}{2} a_{0}+\sum_{m=0}^{\infty} \vec{a}_{m} \cos \left(\frac{m \pi t}{T}\right)+\vec{b}_{m} \sin \left(\frac{m \pi t}{T}\right),
$$

where

$$
\begin{aligned}
\vec{a}_{m} & =\lim _{T \rightarrow \infty} \int_{-T}^{T} \cos \left(\frac{m \pi t}{T}\right) \overrightarrow{\mathcal{E}}_{\perp}(\boldsymbol{r}, t) d t, \\
\vec{b}_{m} & =\lim _{T \rightarrow \infty} \int_{-T}^{T} \sin \left(\frac{m \pi t}{T}\right) \overrightarrow{\mathcal{E}}_{\perp}(\boldsymbol{r}, t) d t,
\end{aligned}
$$

and $a_{0}=0$. For the numerical calculations we take a large period $T=20 \mathrm{ps}$. The intensity spectrum of oscillations is

$$
I\left(\omega_{m}\right) \propto \sum_{m}\left(\vec{a}_{m}^{2}+\vec{b}_{m}^{2}\right)
$$

The plot of $I\left(\omega_{m}\right)$ is given in Fig. [6] The strongest frequency peak corresponds to oscillations with the basic frequency $\omega=\Omega$. The peaks on the high frequency side correspond to the interband excitations and are characteristic of ZB. The peaks on the lower frequency side correspond to the intraband (cyclotron) excitations. At higher magnetic fields we may expect smaller number of distinct frequencies, while for lower fields the classical radiation will evolve toward a quasi-continuous spectrum. In absence of Zitterbewegung the emission spectrum would contain only the intraband (cyclotron) frequencies (see Appendix D). Thus the interband frequencies $\omega_{n}^{Z}$ shown in Fig. [6 are a direct signature of the trembling motion. It can be seen that the $\omega_{n}^{Z}$ peaks are not drastically weaker than the central peak at $\omega=\Omega$ which means that there exists a reasonable chance to observe them.

In Fig. 7 we plot the dependence of the emitted power intensity on the initial wave vector $k_{0 x}$ for three lines (calculated for $B=10 \mathrm{~T}$ ): the basic cyclotron line at $\omega=\Omega$, the intraband frequency $\omega_{3}^{c}$, and the interband frequency $\omega_{6}^{Z}$. It is seen that the intensity of various emission lines depends differently on $k_{0 x}$. At small $k_{0 x}$ values the basic line $\omega=\Omega$ dominates, but at $k_{0 x} \simeq 0.04 \AA^{-1}$ the intensities of various lines become comparable. The characteristic two maxima of $\omega_{6}^{Z}$ occur also for the other interband frequencies. We believe that the $k_{0 x}$ dependence of the line intensities, as shown in Fig. 77, can serve as a signature of ZB.

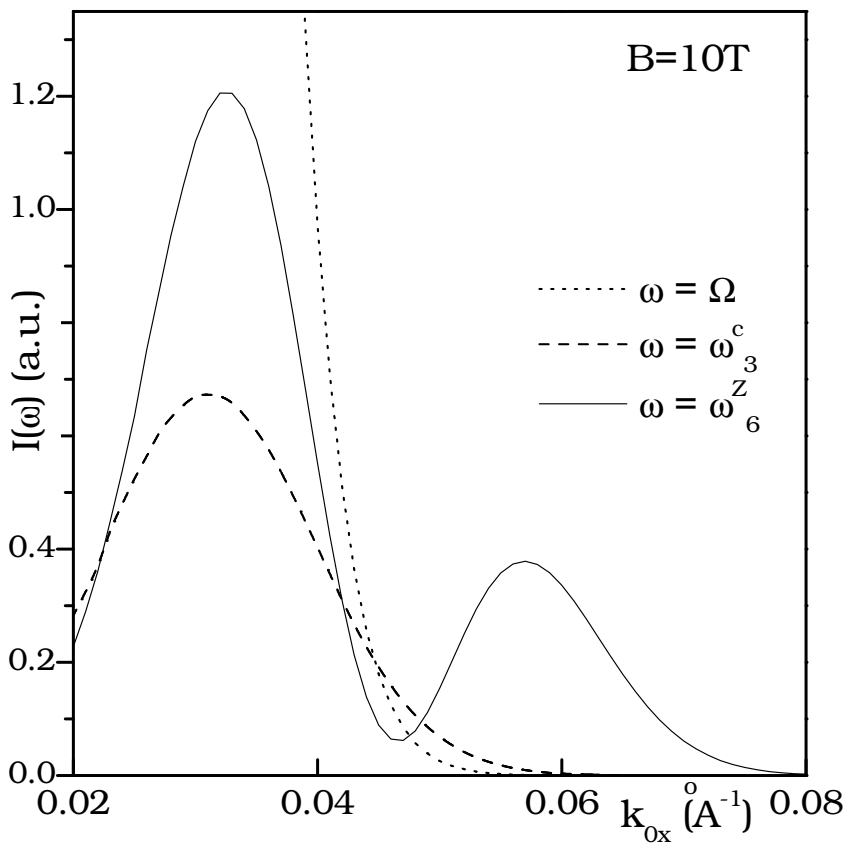

FIG. 7: Intensities of emission lines corresponding to $\omega_{3}^{c}$ (dashed line), $\omega_{0}=\Omega$ (dotted line) and to $\omega_{6}^{Z}$ (solid line) versus the wave vector $k_{0 x}$ of Gaussian wave packet at $B=10$ $\mathrm{T}$.

The properties shown in Figs. 2- 7 have been calculated for the $K_{1}$ point of the Brillouin zone in graphene. The main features applying to the $K_{2}$ point of BZ are described in Appendix E. The above calculations are somewhat idealized since they do not take into account the position of the Fermi energy in a given sample. Thus they correspond approximately to a situation with the Fermi energy relatively low in the valence band. Clearly, the frequencies corresponding to transitions with the final states below the Fermi energy are not possible.

In general terms, the excitation of the system we propose is due to the nonzero momentum $\hbar k_{0 x}$ given to the electron. It can be provided by accelerating the electron in the band by or by exciting the electron with a nonzero momentum by light from the valence band to the conduction band. The electron can emit light because the Gaussian wave packet is not an eigenstate of the system described by Hamiltonian (11). The energy of the emitted light is provided by the initial kinetic energy related to the momentum $\hbar k_{0 x}$. Once this energy is completely used the emission will cease. If the electron is described by a non-gaussian wave packet, all our results are quantitatively valid, only the intensity spectrum will differ from that shown in Fig. 6. We emphasize that the sustained character of ZB oscillations caused by a discrete energy spectrum makes graphene in a magnetic field probably the most favorable system for an experimental observation of the trembling motion considered until present. 


\section{SUMMARY}

We described the Zitterbewegung of electrons in solids in the presence of a magnetic field assuming that the electrons are represented by Gaussian wave packets. The system under consideration is monolayer graphene. It is shown that the presence of a quantizing magnetic field has a profound influence on the Zitterbewegung. In particular, the discrete energy spectrum in a magnetic field causes the Zitterbewegung to be sustained in time while for $B=0$ the $\mathrm{ZB}$ has a transient character. In addition, at $B \neq 0$ many $\mathrm{ZB}$ frequencies appear whereas at $B=0$ one deals with only one ZB frequency. For a given value of initial electron momentum, the magnetic field intensity affects not only ZB frequencies but the entire shape of the ZB spectrum. We consider and describe an electromagnetic radiation emitted by the trembling electrons. Graphene in a magnetic field seems to be a very favorable system for an experimental observation of Zitterbewegung.

\section{APPENDIX A}

We consider a sum rule for the coefficients $U_{m, n}^{\alpha, \beta}$ of Eq. (20). Let us calculate $1=\langle f \mid f\rangle$,

$$
\begin{aligned}
1 & =\sum_{\mathrm{n}}|\langle f \mid \mathrm{n}\rangle|^{2}=\sum_{n, s} \int d k_{x}\left|-s F_{n-1}^{u}\left(k_{x}\right)+F_{n}^{l}\left(k_{x}\right)\right|^{2} \\
& =2 \sum_{n=0}\left(U_{n, n}^{u, u}+U_{n, n}^{l, l}\right) .
\end{aligned}
$$

Factor 2 appears due to the summation over $s$. It is to be reminded that $a_{u}^{2}+a_{l}^{2}=1$. The above sum rule can be used for a verification of the numerical values of $U_{n, n}^{\alpha, \alpha}$.

\section{APPENDIX B}

We calculate $\bar{v}_{y}(t)$ from Eq. (17) for a situation when $U_{n, n}^{\alpha, \beta}$ have a maximum for a large value of $n$. In our model there is $U_{n, n}^{\alpha, \beta}=a_{\alpha} a_{\beta} U_{n, n}$. We use the Poisson summation formula for an estimation of the velocity average disregarding $a_{\alpha}$ and $a_{\beta}$ coefficients. Upon replacing $U_{m, n}$ by a continuous variable $U(x)$ and approximating $U_{n, n \pm 1}=U_{n \pm 1, n} \approx U(x)$, the term with $V_{n}^{-}$in Eq. (17) vanishes. Then

$$
\begin{array}{r}
\bar{v}_{y}(t) \approx 4 u \int_{0}^{\infty} \sin \left(\omega_{x} t\right) U(x) d x \\
+4 u \sum_{l=1}^{\infty} \int \sin \left(\omega_{x} t\right) U(x) \cos (2 \pi x l) d x,
\end{array}
$$

where $\omega_{x}=\Omega(\sqrt{x+1}-\sqrt{x})$. For sufficiently small times we may disregard the second term and $\bar{v}_{y}(t)$ is given by the first integral in Eq. (B1). In Fig. 8 we show the results of the integration compared with the exact

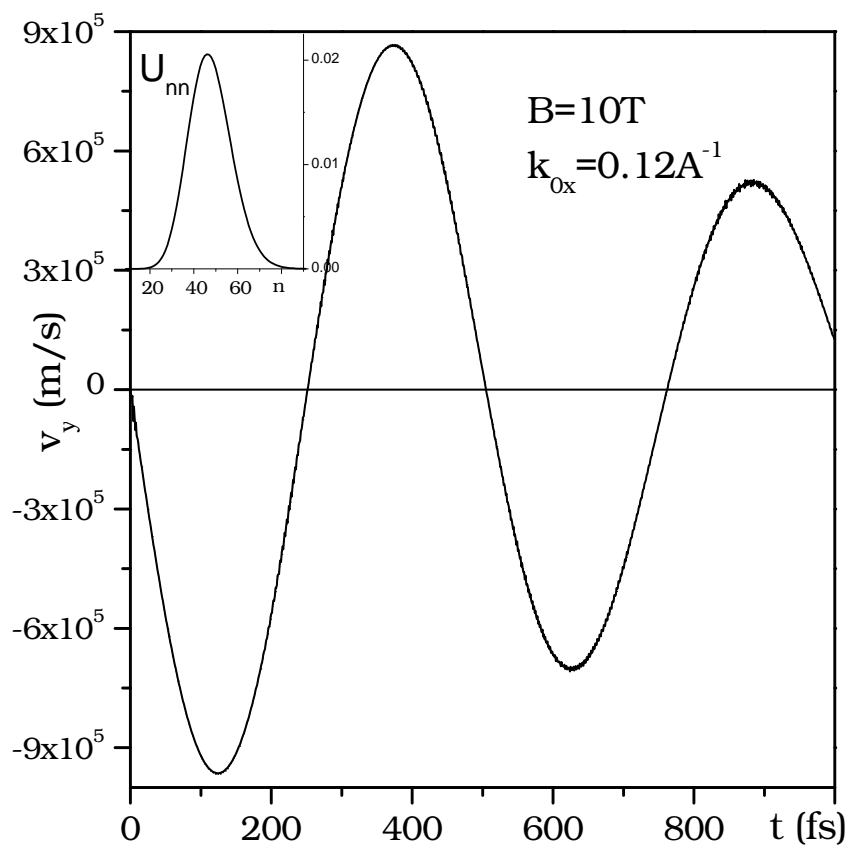

FIG. 8: Contribution of the $K_{1}$ point to the electron velocity $\bar{v}_{y}(t)$ versus time, calculated for the indicated parameters: (a) using full formula (17) (see Fig. 2d), (b) using the first integral of the Poisson formula (B1). The two curves practically coincide. Inset shows coefficients $U_{n, n \pm 1}=U_{n \pm 1, n} \approx U(x)$ for $k_{0 x}=0.12 \AA^{-1}$. The frequency of oscillations corresponds to $\omega_{\max }$ for $x_{\max }=46$, see text.

calculations of Eq. (17) for $k_{0 x}=0.12 \AA^{-1}$. The two curves practically coincide, apart from the small contributions of higher ZB frequencies present in exact formula (17). The effective frequency of the motion is given by $\omega_{\max }=\Omega\left(\sqrt{x_{\max }+1}-\sqrt{x_{\max }}\right)$, where $x_{\max }=46$ corresponds to the maximum of $U(x)$, see inset. For larger times the second term in Eq. (B1) is not negligible and full formula (B1) is equivalent to Eq. (17).

\section{APPENDIX C}

Here we consider briefly the gauge aspects. According to the general theory [44], if one introduces a new gauge by means of an arbitrary function $\Lambda(\boldsymbol{r})$, the new vector potential is $\boldsymbol{A}^{\prime}=\boldsymbol{A}+\boldsymbol{\nabla} \Lambda$, and the new scalar potential is $A_{0}^{\prime}=A_{0}+\partial \Lambda / \partial t$. Then the wave function changes as $\Psi^{\prime}=e^{(i e / \hbar) \Lambda} \Psi$, and the gauge invariance for an operator $\hat{O}=\hat{O}\left(\boldsymbol{A}, A_{0}\right)$ means

$$
\left\langle\Psi\left|\hat{O}\left(\boldsymbol{A}, A_{0}\right)\right| \Psi\right\rangle=\left\langle\Psi^{\prime}\left|\hat{O}\left(\boldsymbol{A}^{\prime}, A_{0}^{\prime}\right)\right| \Psi^{\prime}\right\rangle .
$$

This leads to

$$
\begin{aligned}
& \hat{O}\left(\boldsymbol{A}^{\prime}, A_{0}^{\prime}\right)=e^{(i e / \hbar) \Lambda} \hat{O}\left(\boldsymbol{A}, A_{0}\right) e^{-(i e / \hbar) \Lambda}= \\
& \hat{O}\left(\boldsymbol{A}, A_{0}\right)+\left[e^{(i e / \hbar) \Lambda}, \hat{O}\left(\boldsymbol{A}, A_{0}\right)\right] e^{-(i e / \hbar) \Lambda} .
\end{aligned}
$$

If, instead of the gauge $\boldsymbol{A}=(-B y, 0,0)$, we take $\boldsymbol{A}^{\prime}=$ $(0, B x, 0)$, which gives the same magnetic field, we have 
$\Lambda=x y / L^{2}$, so that $\Psi^{\prime}(x, y)=e^{i x y / L^{2}} \Psi(x, y)$. Using prescription (C2) and calculating

$$
\begin{aligned}
& {\left[e^{i x y / L^{2}}, \hat{p}_{x}\right]=-e B y e^{i x y / L^{2}},} \\
& {\left[e^{i x y / L^{2}}, \hat{p}_{y}\right]=-e B x e^{i x y / L^{2}},}
\end{aligned}
$$

one shows that relation (C1) is satisfied also for Hamiltonian (1) in the new gauge.

\section{APPENDIX D}

We consider here the motion of a wave packet in the presence of a magnetic field according to the Schrodinger equation. For 2D Hamiltonian there is $|\mathrm{n}\rangle=\left|n k_{x}\right\rangle=$ $e^{i k_{x} x} \mathrm{H}_{\mathrm{n}}(\xi) e^{-\xi^{2} / 2} /\left(\sqrt{2 \pi L} C_{n}\right)$, and $E_{n}=\hbar \omega_{c}(n+1 / 2)$, where $\omega_{c}=e B / m$. The velocity average $\bar{v}_{x}(t)$ is

$\bar{v}_{x}(t)=\frac{1}{m_{n, n^{\prime}, k_{x}, k_{x}^{\prime}}}\left\langle f \mid n^{\prime} k_{x}^{\prime}\right\rangle\left\langle n k_{x} \mid f\right\rangle e^{i \omega_{c}\left(n^{\prime}-n\right) t}\left\langle n^{\prime} k_{x}^{\prime}\left|\hat{\pi}_{x}\right| n k_{x}\right\rangle$,

and similarly for $\bar{v}_{y}(t)$. Since

$\left\langle n^{\prime} k_{x}^{\prime}\left|\hat{\pi}_{x}\right| n k_{x}\right\rangle=-\frac{\hbar \delta_{k_{x}, k_{x}^{\prime}}}{\sqrt{2} L}\left(\sqrt{n} \delta_{n^{\prime}, n-1}+\sqrt{n+1} \delta_{n^{\prime}, n+1}\right)$,

we have

$$
\begin{aligned}
\bar{v}_{x}(t) & =-\frac{\hbar}{\sqrt{2} L m} \int d k_{x} \sum_{n=1}^{\infty}\langle f \mid n-1\rangle\langle n \mid f\rangle \sqrt{n} e^{-i \omega_{c} t}+ \\
& -\frac{\hbar}{\sqrt{2} L m} \int d k_{x} \sum_{n=0}^{\infty}\langle f \mid n+1\rangle\langle n \mid f\rangle \sqrt{n+1} e^{i \omega_{c} t} .
\end{aligned}
$$

There is $\sqrt{n}|n-1\rangle=\hat{a}|n\rangle$ and $\sqrt{n+1}|n+1\rangle=\hat{a}^{+}|n\rangle$, and we calculate

$$
\bar{v}_{x}(t)=\frac{\hbar}{\sqrt{2} L m} \int d k_{x}\left(\langle f \hat{a} \mid f\rangle e^{-i \omega_{c} t}+\left\langle f \hat{a}^{+} \mid f\right\rangle e^{i \omega_{c} t}\right),
$$

and similarly for $\bar{v}_{y}(t)$. For a one-component wave packet of Eq. (21) the integrals indicated in Eq. (D4) can be done analytically. We finally obtain

$$
\begin{aligned}
& \bar{v}_{x}(t)=\frac{\hbar k_{0 x}}{2 m}\left(-e^{-i \omega_{c} t}-e^{i \omega_{c} t}\right)=-\frac{\hbar k_{0 x}}{m} \cos \left(\omega_{c} t\right), \\
& \bar{v}_{y}(t)=i \frac{\hbar k_{0 x}}{2 m}\left(e^{-i \omega_{c} t}-e^{i \omega_{c} t}\right)=-\frac{\hbar k_{0 x}}{m} \sin \left(\omega_{c} t\right)(\mathrm{D} 5)
\end{aligned}
$$

Thus an electron represented by a Gaussian wave packet having the initial momentum $\hbar k_{0 x}$ moves on a circular orbit with the cyclotron frequency $\omega_{c}$ without attenuation. A similar result is known for a one-dimensional wave packet moving in a parabolic potential. On the other hand, the motion illustrated in Fig. 2d is damped during the first picosecond which is an another manifestation of Zitterbewegung.

\section{APPENDIX E}

Here we consider contributions related to ZB of electrons at the inequivalent point $K_{2}$ of the Brillouin zone. The form of Hamiltonian at the $K_{2}$ point is somewhat controversial, various authors give different expressions. According to Refs. [45] and [46] the Hamiltonian $\hat{H}^{\prime}$ is

$$
\hat{H}^{\prime}=u\left(\begin{array}{cc}
0 & -\hat{\pi}_{x}-i \hat{\pi}_{y} \\
-\hat{\pi}_{x}+i \hat{\pi}_{y} & 0
\end{array}\right)
$$

i.e. $\hat{H}^{\prime}=-\hat{H}^{T}$. The eigenvectors of $\hat{H}^{\prime}$ are

$$
\left|n k_{x} s\right\rangle^{\prime}=\frac{e^{i k_{x} x}}{\sqrt{4 \pi}}\left(\begin{array}{c}
|n\rangle \\
s|n-1\rangle
\end{array}\right) .
$$

i.e. they differ from those given by Eq. (91). The quantum velocity $\partial \hat{H}^{\prime} / \partial \hat{p}_{x}=-u \sigma_{x}$ and $\partial \hat{H}^{\prime} / \partial \hat{p}_{y}=+u \sigma_{y}$. Thus, the $x$ component of the velocity changes sign, while the $y$ component remains unchanged. Repeating the calculations we obtain again Eqs. (17) and (18), in which the coefficients are

$$
\begin{aligned}
& \tilde{V}_{n}^{ \pm}=-U_{n, n+1}^{u, u}-U_{n+1, n}^{u, u} \mp U_{n, n-1}^{l, l} \mp U_{n-1, n}^{l, l}, \\
& \tilde{T}_{n}^{ \pm}=+U_{n, n+1}^{u, u}-U_{n+1, n}^{u, u} \mp U_{n, n-1}^{l, l} \pm U_{n-1, n}^{l, l}, \\
& \tilde{A}_{n}^{ \pm}=-U_{n, n}^{u, l}+U_{n, n}^{l, u} \pm U_{n+1, n-1}^{u, l} \mp U_{n-1, n+1}^{l, u}, \\
& \tilde{B}_{n}^{ \pm}=-U_{n, n}^{u, l}-U_{n, n}^{l, u} \mp U_{n+1, n-1}^{u, l} \mp U_{n-1, n+1}^{l, u} .
\end{aligned}
$$

If we are interested in the electric current, we should add the velocities of the two inequivalent points of the BZ. As a consequence, the $x$ component of the velocity vanishes while the $y$ component nearly doubles.
[1] E. Schrodinger, Sitzungsber. Preuss. Akad. Wiss. Phys. Math. Kl. 24, 418 (1930). Schrodinger's derivation is reproduced in A. O. Barut and A. J. Bracken, Phys. Rev. D 23, 2454 (1981).

[2] F. Cannata, L. Ferrari, and G. Russo, Sol. St. Comun. 74, 309 (1990); L. Ferrari and G. Russo, Phys. Rev. B
42, 7454 (1990).

[3] N. Shmueli, A. Yacoby, and Y. Imry, Europhys. Lett. 29, 711 (1995).

[4] J. Schliemann, D. Loss, and R. M. Westervelt, Phys. Rev. Lett. 94, 206801 (2005).

[5] W. Zawadzki, Phys. Rev. B 72, 085217 (2005). 
[6] W. Zawadzki, Phys. Rev. B 74, 205439 (2006).

[7] J. Cserti and G. David, Phys. Rev. B 74, 172305 (2006).

[8] M. I. Katsnelson, Europ. Phys. J. B 51, 157 (2006).

[9] R. Winkler, U. Zulicke, and J. Bolte, Phys. Rev. B 75, 205314 (2007).

[10] B. Trauzettel, Y. M. Blanter, and A. F. Morpurgo, Phys. Rev. B 75, 035305 (2007).

[11] T. M. Rusin and W. Zawadzki, J. Phys. Cond. Matter 19, 136219 (2007).

[12] T. M. Rusin and W. Zawadzki, Phys. Rev. B 76, 195439 (2007).

[13] U. Zulicke, J. Bolte, and R. Winkler, New J. Phys. 9, 355 (2007).

[14] W. Zawadzki, in Optical Properties of Solids, edited by E. D. Haidemenakis (Gordon and Breach, New York, 1970), p. 179.

[15] W. Zawadzki, in High Magnetic Fields in the Physics of Semiconductors II, edited by G. Landwehr and W. Ossau (World Scientific, Singapore, 1997), p. 755.

[16] J. A. Lock, Am. J. Phys. 47, 797 (1979).

[17] W. Zawadzki and T. M. Rusin, Preprint arXiv:condmat/0805.0478 (2008).

[18] K. Huang, Am. J. Phys. 20, 479 (1952).

[19] B. M. Garraway and K. A. Suominen, Rep. Prog. Phys. 58, 365 (1995).

[20] J. Y. Vaishnav and C. W. Clark, Preprint arXiv:condmat/0711.3270 (2007).

[21] M. Merkl, F. E. Zimmer, G. Juzeliunas, and P. Ohberg, Preprint arXiv:cond-mat/0803.4189 (2008).

[22] X. Zhang, Phys. Rev. Lett. 100, 113903 (2008).

[23] A. Bermudez, M. A. Martin-Delgado, and A. Luis, Phys. Rev. A 77, 033832 (2008).

[24] X. Zhang and Z. Liu, Preprint arXiv:cond-mat/0804.1978 (2008).

[25] T. M. Rusin and W. Zawadzki, Preprint arXiv:condmat/0712.3590 (2007).

[26] J. Schliemann, New J. Phys. 10, 034024 (2008).

[27] K. S. Novoselov, A. K. Geim, S. V. Morozov, D. Jiang, Y. Zhang, S. V. Dubonos, I. V. Grigorieva, and A. A.
Firsov, Science 306, 666 (2004).

[28] K. S. Novoselov, A. K. Geim, S. V. Morozov, D. Jiang, M. I. Katsnelson, I. V. Grigorieva, S. V. Dubonos, and A. A. Firsov, Nature 438, 197 (2005).

[29] A. K. Geim and K. S. Novoselov, Nature Materials 6, 183 (2007).

[30] P. R. Wallace, Phys. Rev. 71, 622 (1947).

[31] J. C. Slonczewski and P. R. Weiss, Phys. Rev. 109, 272 (1958).

[32] Y. Zhang, Y. W. Tan, H. L. Stormer, and P. Kim, Nature 438, 201 (2005).

[33] M. L. Sadowski, G. Martinez, M. Potemski, C. Berger, and W. A. de Heer, Phys. Rev. Lett. 97, 266405 (2006).

[34] J. D. Bjorken and S. D. Drell, Relativistic Quantum Mechanics (McGraw-Hill, New York, 1964).

[35] W. Greiner, Relativistic Quantum Mechanics (Springer, Berlin, 1994).

[36] A. P. Prudnikov, J. A. Brychkov, and O. I. Marichev, Integrals and Series (Fizmatlit, Moscow, 2003).

[37] C. C. Gerry and P. L. Knight, Introductory Quantum Optics, (Cambridge Univ. Press, Cambridge 2005).

[38] E. T. Jaynes and F. W. Cummings, Proc, IEEE 51, 89 (1963).

[39] G. Rempe, H. Walther, and N. Klein, Phys. Rev. Lett. 58, 353 (1987).

[40] M. A. Topinka, B. J. LeRoy, S. E. J. Shaw, E. J. Heller, R. M. Westervelt, K. D. Maranowski, and A. C. Gossard, Science 289, 2323 (2000).

[41] B. J. LeRoy, J. Phys. Cond. Matter 15, R1835 (2003).

[42] D. Bohm, Quantum Theory (Prentice-Hall, New York, 1952).

[43] J. D. Jackson, Classical Electrodynamics, (John Wiley \&. Sons, New York, 1975).

[44] D. Kobe and A. Smirl, Am. J. Phys. 46, 633 (1978).

[45] V. P. Gusynin, S. G. Sharapov, and J. P. Carbotte, Preprint arXiv:cond-mat/0706.3016 (2007).

[46] C. Bena and G. Montambaux, Preprint arXiv:condmat/0712.0765 (2007). 